\section{Kendall, Edward Calvin}

\section{W. Hubl}

Ehem. Krankenhaus Dresden-Friedrichstadt, Institut für Klinische Chemie und Labormedizin, Dresden, Deutschland

Lebensdaten US-amerikanischer Biochemiker, geboren am 8. März 1886 in South Norwalk (Connecticut, USA), gestorben am 4. Mai 1972 in Princeton (New Jersey, USA). Kendall wurde im Jahr 1910 Ph.D im Fach Chemie. an der Columbia Universität, 1910-1914 begann er mit seinen Forschungsarbeiten zur Wirkungsweise der Schilddrüse am St. Luke's Hospital in New York City. Im Jahr 1914 übernahm er die Leitung der Abteilung für Biochemie an der Mayo Klinik und erhielt 1921 eine Professur für Physiologische Chemie an der Mayo Foundation (Universität von Minnesota). Hier gelang ihm 1915 die Isolierung und Aufklärung der chemischen Struktur von Thyroxin sowie von Glutathion.

Verdienste Kendalls wichigste Entdeckung war im Jahr 1935 die Isolierung des Steroidhormones Kortison ( $\triangleright$ Steroidhormone) aus der Nebennierenrinde, das von ihm ursprünglich als ,,compound E“ bezeichnet wurde. Zwei weitere
Arbeitsgruppen isolierten Kortison in rascher Folge und bezeichneten dieselbe Substanz als „,compound F“ durch Wintersteiner und Substanz $\mathrm{Fa}$ durch Reichstein ( $\triangleright$ Reichstein, Tadeus). Gemeinsam mit Philip Showalter Hench setzte er im Jahr 1948 dieses Nebennierenrindenhormon erstmals zur entzündungshemmenden Behandlung der rheumatoiden Arthritis mit großartigem Erfolg ein. 1951 beendete Kendall altershalber seine Tätigkeit an der Mayo Foundation. Ab 1952 arbeitete er als Visiting Professor für Chemie in der Abteilung für Biochemie an der Universität von Princeton. Nobelpreis für Medizin im Jahr 1950 gemeinsam mit Tadeus Reichstein und Philip S. Hench: „Für die Entdeckungen bei den Hormonen der Nebennierenrinde, ihrer Struktur und ihrer biologischen Wirkungen.“

\section{Literatur}

Herder WW (2014) Heroes in endocrinology: Nobel Prizes. Endocr Connect 3:R98

Ingle DJ (1975) Biographical memoir of Edward C. Kendall, Bd 47. National Academy of Sciences, Washington, DC

Kendall EC, Osterberg AE (1919) The chemical identification of thyroxin. J Biol Chem 40:265-334

Raju TN (1999) The Nobel chronicles. 1950: Edward Calvin Kendall (1886-1972); Philip Showalter Hench (1896-1965); and Tadeus Reichstein (1897-1996). Lancet 353(9161):1370 